\title{
Avifauna del centro forestal tropical Pedro Antonio Pineda, cuenca baja del río Calima (Buenaventura, Colombia)
}

\author{
Birds of the "Pedro Antonio Pineda" tropical forest center, \\ lower basin of Calima river (Buenaventura, Colombia)
}

\author{
Stefanny Patiño-Forero' ${ }^{\circledR}$, Camila Martínez-Páez ${ }^{\circledR}{ }^{(}$, Miguel Ángel Quimbayo-Cardona $^{(}$ \\ y Hugo Nelson Loaiza-Hernández ${ }^{2}$
}

Recepción: 27 de abril de 2019

Aprobación: 21 de mayo de 2020

Patiño-Forero, S., Martínez-Páez, C., Quimbayo-Cardona, M. A. y Loaiza-Hernández, H. N. (2020). Avifauna del centro forestal tropical Pedro Antonio Pineda, cuenca baja del río Calima (Buenaventura, Colombia). Colombia Forestal, 23(2), 59-74.

\section{Resumen}

El Centro Forestal Tropical (CFT) Pedro Antonio Pineda (Buenaventura, Colombia) es el principal centro rural de formación académica de la Facultad de Ingeniería Forestal de la Universidad del Tolima, el cual se ubica en territorio del consejo comunitario de comunidades afrodescendientes. Entre 2013 y 2017 se realizaron observaciones en siete localidades asociadas a tres tipos de hábitat (áreas abiertas, borde de bosque e interior de bosque), principalmente en abril y septiembre para aportar al conocimiento de las aves del bosque húmedo tropical del CFT. Se registraron 166 especies (20 órdenes y 40 familias taxonómicas); el orden con mayor abundancia relativa fue Passeriformes (62.6 \%) y las familias con mayor abundancia relativa fueron Thraupidae $(17.3 \%)$ y Tyrannidae $(8.9 \%)$. Se registraron 22 especies casi endémicas y 31 con estatus migratorio; desde un contexto regional la mayoría de las aves fueron registradas en áreas abiertas (44 especies) seguidas por especies de borde de bosque (42 especies).

Palabras clave: abundancia, bajo Calima, biodiversidad, bosque húmedo tropical, endemismos.

\begin{abstract}
The Tropical Forestry Center (CFT) "Pedro Antonio Pineda" (Buenaventura, Colombia), is the main academic training center with rural character at the Faculty of Forestry Engineering, University of Tolima, located at the territory of the community council of Afro-descendant communities. Between 2013 and 2017, observations were made in seven locations associated with three habitat types (open areas, forest edge and forest interior), mainly in April and September, to provide knowledge of the tropical forest birds of the CFT. In overall, 166 species were registered (20 orders and 40 taxonomic families); the order with the highest relative abundance was Passeriformes $(62.6 \%)$ and the families with the highest relative abundance were Thraupidae $(17.3 \%)$ and Tyrannidae (8.9\%). There were 22 species nearly endemic and 31 with migratory status; from a regional context, most of the birds were recorded in open areas (44 species) followed by forest edge species (42 species).
\end{abstract}

Keywords: abundance, bajo Calima, biodiversity, endemism, tropical humid forest.

Universidad del Tolima. Ibagué, Colombia. spatinof@ut.edu.co; martinezkami@hotmail.com; miguelq@ut.edu.co; hnoaiza.h@ut.edu.co Universidad del Tolima, Ibagué, Colombia. hnoaiza.h@ut.edu.co 


\section{INTRODUCCIÓN}

Colombia forma parte de los 14 países biológicamente más ricos del mundo. Ocupa el $0.22 \%$ de la superficie terrestre y se posiciona en el segundo lugar en biodiversidad, sobresaliendo por albergar el $10 \%$ del total de la fauna y flora existentes en la tierra en una extensión del $0.8 \%$ de la superficie del planeta (Andrade, 2011; Instituto de Investigación de Recursos Biológicos Alexander von Humboldt, 2012). En cuanto a aves, sobresale por ser el país más diverso ya que posee alrededor de 1932 especies, equivalente al $19 \%$ de las 10507 especies de aves conocidas en el mundo, superando en diversidad a países como Perú y Brasil (Ayerbe, 2018; Donegan et al., 2015).

Del total de especies de aves presentes en Colombia existen en riesgo de extinción 118 en los bosques húmedos de los Andes y en la costa Pacífica, de las cuales 87 son endémicas de nuestro territorio nacional (Rengifo et al., 2014). Entre las principales causas de amenazas se encuentran la deforestación, las actividades agrícolas y ganaderas, el comercio y cacería, la extracción de madera y los cultivos ilícitos, entre otros (Andrade, 2011).

Actualmente la pérdida de bosques avanza a un ritmo acelerado. No obstante, en el mundo, en algunos países la deforestación se ha hecho más lenta; en América del Sur se registra la mayor pérdida neta de bosques y en Colombia no se da el caso contrario (Andrade, 2011). Tal situación no ha sido afrontada y la alteración de la cobertura boscosa aumenta continuamente, especialmente en zonas donde no hay o es poco el registro existente sobre su biodiversidad. En los últimos 25 años el país perdió 8.4 \% de los bosques que estaban en pie en 1990, esto a causa principalmente de la tasa de deforestación (García, 2014). El principal determinante de deforestación en Colombia es la conversión de los bosques en tierras agrícolas o ganaderas, la tala ilegal, así como causas naturales como consecuencia del abandono de las prácticas agrícolas tradicionales, entre otras causas (Andrade, 2011; Armenteras y Rodríguez, 2014; García, 2014). En la región Pacífica la principal causa de deforestación y degradación forestal se relaciona con el crecimiento de la industria maderera y la tala ilegal. En esta región se extrae gran parte de la madera aserrada y de la materia prima para la industria de pulpa de papel que se consume en el país; de igual forma, se presenta la transformación de la tierra para cultivos para el autoconsumo y pastos para actividades ganaderas extensivas (García, 2014). Es en tierras bajas donde en la actualidad se presenta la mayor pérdida por deforestación (Armenteras y Rodríguez, 2014).

La costa pacífica colombiana, incluida dentro de la provincia biogeográfica Chocó-Magdalena, es considerada como una de las regiones con mayor diversidad biológica del planeta. Contiene el $8 \%$ de los bosques naturales del país y la mitad de su territorio está constituida por formaciones boscosas húmedas, en las cuales se observa como cobertura dominante los manglares, pastizales húmedos, humedales, bosques aluviales y bosques húmedos de tierras bajas, entre otros (Botero, 2010; Valoyes et al., 2012; García, 2014). Esta región es considerada uno de los más importantes hotspots identificados en el planeta (Chocó-Darién) (Myers et al., 2000), en el cual se tienen registros de 778 especies de aves, 180 especies de mamíferos, 188 especies de reptiles y 137 especies de anfibios (Rangel, 2015).

El Centro Forestal Tropical (CFT) Pedro Antonio Pineda, localizado en el municipio de Buenaventura, se ha caracterizado desde hace más de 40 años por la realización de actividades académicas, de proyección social y de investigación por parte de la Facultad de Ingeniería Forestal de la Universidad del Tolima, enfocadas a estudios del bosque y especies arbóreas que lo componen. En estas se cuenta con la participación directa de las comunidades afrodescendientes de la región de la cuenca baja del río Calima y se orientan al manejo sostenible del bosque, aunque con vacíos sobre el conocimiento de la fauna silvestre, especialmente el grupo de las aves. Para este caso fueron registradas 166 especies de aves que utilizan los bosques secundarios de las cuales el $82 \%$ son residentes y el $18 \%$ son migratorias, incluidas especies en categorías de riesgo y casi endémicas para Colombia. 
El objetivo de este estudio fue conocer la diversidad de la avifauna del bosque húmedo del CFT Pedro Antonio Pineda, en la cuenca bajo del río Calima (Buenaventura, Colombia).

\section{MATERIALES Y MÉTODOS}

\section{Área de estudio}

La región del Bajo Calima cuenta con una extensión que supera las 65000 ha localizadas en la cuenca baja del río Calima; se ubica aproximadamente a $3^{\circ} 59^{\prime} 00^{\prime \prime}$ de latitud norte y $76^{\circ} 53^{\prime} 76^{\prime \prime}$ de longitud oeste, a $40 \mathrm{~km}$ del municipio de Buenaventura, en el departamento del Valle del Cauca, sobre la vertiente izquierda de la cordillera Occidental con influencias por la zona de confluencia de la corriente intertropical. Se define como una provincia climática cálida perhúmeda, la cual se caracteriza por presentar una temperatura promedio anual superior a $24^{\circ} \mathrm{C}$, alturas que van desde 0 a $600 \mathrm{~m}$ de altitud, precipitación de $7445 \mathrm{~mm}$ anuales, alta humedad relativa mayor de $100 \%$ y brillo solar de acuerdo con la época del año y la latitud (Bocanegra et al., 2009). Esta zona se caracteriza por la presencia de comunidades afrodescendientes, quienes bajo la Ley 70 de 1993 conformaron el concejo comunitario del Bajo Calima (Lozano y González, 2009).

En el CFT Pedro Antonio Pineda, Bajo Calima, se encuentran representados bosques muy húmedos y pluviales; actualmente constituidos por bosques secundarios como resultado de la explotación a tala rasa de madera para transformarla en pulpa por parte de la Concesión Forestal de Pulpapel, entre 1959 a 1993 (Lozano y González, 2009). Esta área tiene una extensión de aproximadamente 20000 ha equivalentes al $35 \%$ del territorio; el resto de vegetación está conformada por bosques primarios afectados por la actividad antrópica de la explotación maderera, clasificándolos como bosques primarios intervenidos a poco intervenidos $(50 \%$ del territorio). Estos bosques se encuentran circunscritos a las áreas del territorio con poca accesibilidad en los paisajes de colinas altas y se estima que representan al menos 15 \% del área total (Moreno, 2009).

\section{Registro de aves}

Entre los años 2013 y 2017 se realizaron observaciones en tres tipos de hábitat (área abierta, borde de bosque e interior de bosque) en los bosques secundarios encontrados en los alrededores del CFT, una vez por semestre durante una semana principalmente en los meses de abril y septiembre con el fin de tomar registros en las dos diferentes temporadas climáticas que se presentan en la región y así poder observar cambios en la presencia, riqueza y abundancia de especies. Las observaciones al interior del bosque se realizaron a $200 \mathrm{~m}$ del borde.

El registro de aves se realizó siguiendo la metodología propuesta por Villareal et al. (2004). Se realizaron observaciones con binóculos $7 \times 50$ en las mañanas entre las 05:30 y 10:00 horas y en las tardes entre las 16:00 y 19:00 horas. Para un total de 630 horas de observación (90 horas por tipo de hábitat), tomando puntos de georreferenciación en cada uno (tabla 1 y figura 1 ).

Tabla 1. Sitios de muestreo para el avistamiento de aves del CFT Pedro Antonio Pineda, Bajo Calima, Buenaventura

\begin{tabular}{|c|c|c|c|c|c|}
\hline \multirow{2}{*}{ Punto conteo } & \multicolumn{2}{|c|}{ Coordenadas } & \multirow{2}{*}{ Localidad } & \multirow{2}{*}{ Tipo de hábitat } & \multirow{2}{*}{ Altura (m) } \\
\hline & $\mathbf{X}$ & $\mathbf{Y}$ & & & \\
\hline 1 & 1009710.907 & 928959.893 & Quebrada el puente & Área abierta & 34 \\
\hline 2 & 1009983.013 & 928795.895 & Quebrada el puente & Área abierta & 37 \\
\hline 3 & 1010433.696 & 927351.406 & Borde carretera (Vía CFT-Vivero) & Borde bosque & 73 \\
\hline 4 & 1009494.326 & 929168.129 & Borde carretera (Vía CFT-Brisas) & Borde bosque & 16 \\
\hline 5 & 1009098.2 & 929426.108 & Borde carretera (Vía CFT-Brisas) & Borde bosque & 57 \\
\hline 6 & 1009629.47 & 928915.653 & CFT & Borde bosque & 18 \\
\hline 7 & 1009592.452 & 928913.806 & Sotobosque (CFT) & Interior bosque & 21 \\
\hline
\end{tabular}




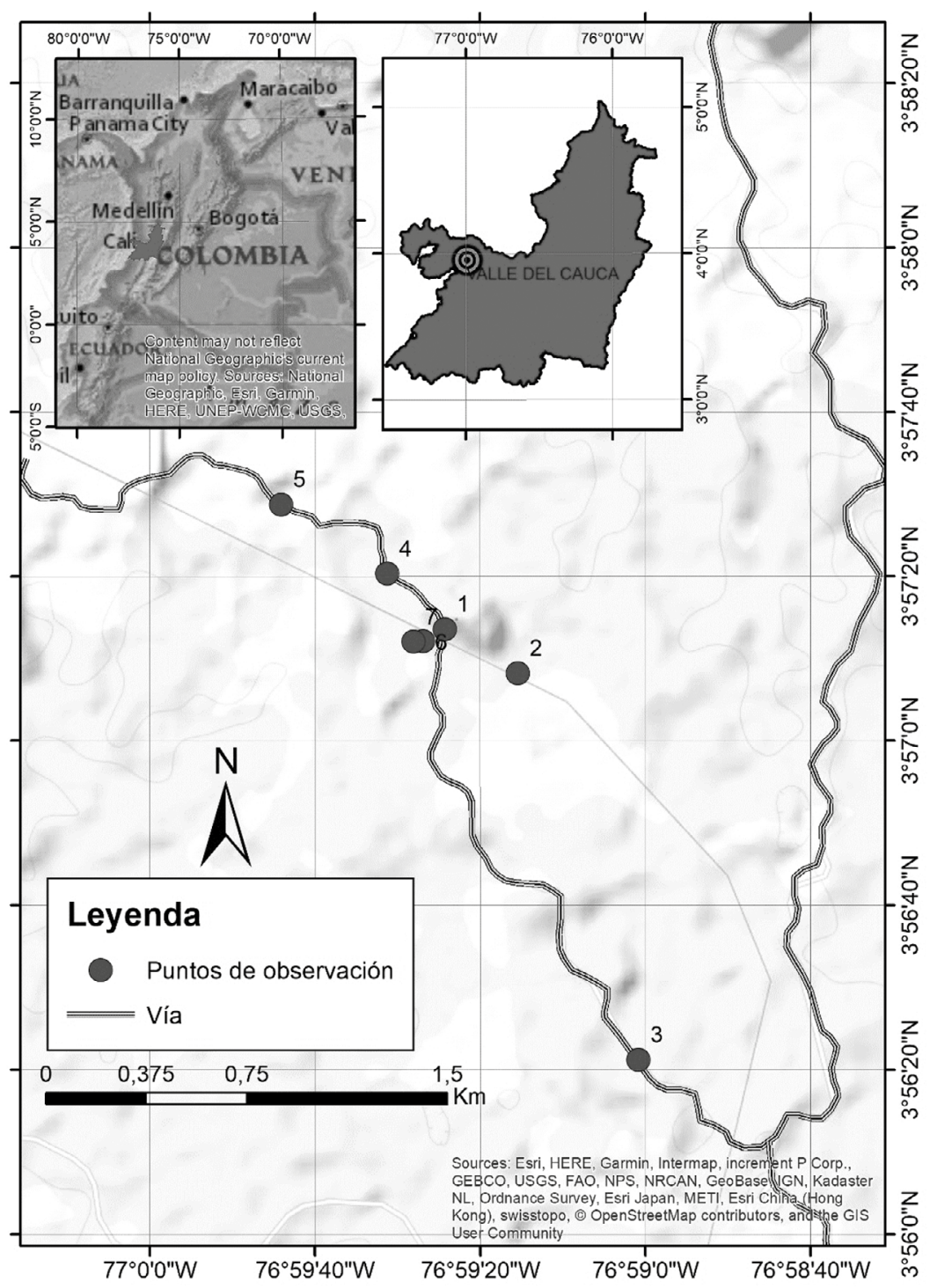

Figura 1. Localización del CFT Pedro Antonio Pineda, Bajo Calima, Buenaventura.

Durante las observaciones en campo se realizaron anotaciones de las especies avistadas (nombre común y científico), número de individuos por especie, tipo de hábitat donde se registró y sexo (para las especies con dimorfismo sexual).

Los individuos observados fueron identificados hasta su especie; para la identificación se utilizaron guías de identificación de aves de Colombia (McMullan et al., 2010). La clasificación taxonómica de las especies siguió la propuesta de South American Classification Committee (versión 8, julio de 2019). Se realizó un listado de aves y se procedió a la verificación de su distribución en la región del Pacífico con base en Ayerbe (2018).

\section{Análisis de los datos}

Para el análisis de las especies de aves registradas fueron tenidos en cuenta aspectos ecológicos, presiones, amenazas y vulnerabilidades, soportados en la documentación de historia de vida de los libros de guía de identificación de aves para 
Colombia. Estos análisis corresponden a rasgos funcionales tales como dieta (D), estrategia de forrajeo $(\mathrm{F})$, estrato de forrajeo (EF), tipo de anidamiento (TA), comportamiento social (CS), estatus migratorio (EM) (López et al., 2016) y se estableció tipo de anidación en cavidad (excavadoras o no excavadoras) (McComb, 2008) y grado de amenaza según Renjifo et al. (2014). Esto se complementó con la determinación de cuáles de las especies son endémicas o casi endémicas según el listado actualizado por Chaparro et al. (2013), Avendaño et al. (2017) y Ayerbe (2018).

Dependiendo del tipo de hábitat se clasificó según las "categorías ecológicas" establecidas por Stiles y Bohórquez (2000), las cuales corresponden a:

"I: especies de bosque, dividas en la: especies restringidas al bosque primario o poco alterado y $\mathrm{lb}$ : especies no restringidas al bosque primario o poco alterado; II: especies de bosque secundario o bordes de bosque, o de amplia tolerancia; III: especies de áreas abiertas, IV: especies acuáticas dividida en dos grupos, IVa: especies acuáticas asociadas a cuerpos de agua sombreados o con la vegetación densa al borde del agua y IVb, especies acuáticas asociadas a cuerpos de agua sin sombra, orillas abiertas o con vegetación baja; y por último $\mathrm{V}$ : especies aéreas dividas en $V a$ : especies aéreas que requieren por lo menos parches de bosque; $\mathrm{Vb}$, especies aéreas indiferentes a la presencia de bosque".

\section{RESULTADOS}

Se reconoció un total de 562 individuos, pertenecientes a 166 especies de 20 órdenes y 40 familias taxonómicas (anexo 1), los cuales fueron registrados mediante observación directa. El orden de mayor abundancia relativa fue Passeriformes (62.5\%), seguido de Apodiformes (8.3\%). La familia de mayor abundancia relativa de especies correspondió a Thraupidae (17.3\%), seguida de las familias Tyrannidae $(8.9 \%)$, Trochillidae $(7.1 \%)$ y Thamnophilidae $(6.5 \%)$ y otras con menor abundancia relativa (figura 2 ).

La mayoría de las aves registradas (45\%) se consideraron como exclusivamente insectívoras, $30 \%$ fueron frugívoras y $8 \%$ granívoras y nectarívoras, respectivamente (figura 3). Con base en información de la historia de vida de las especies referenciada en Hilty y Brown (1986) y en las

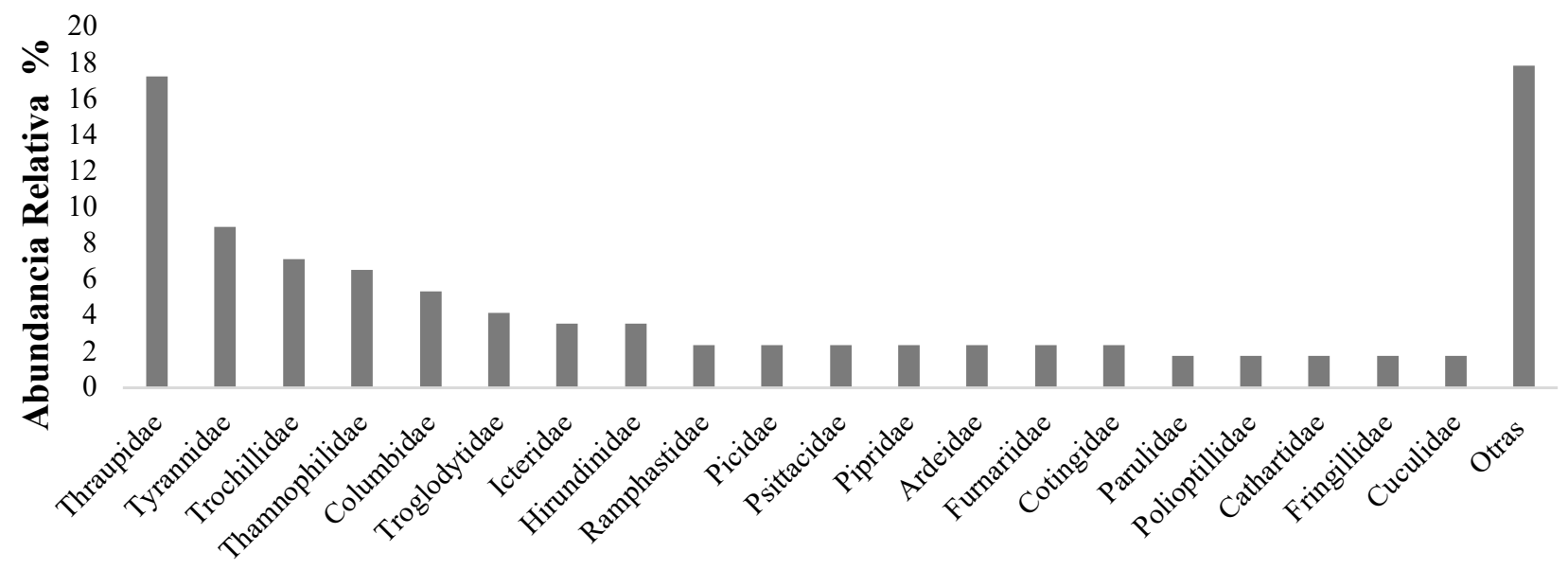

Familia

Figura 2. Abundancia relativa de especies de aves por familia, para los alrededores y en CFT Pedro Antonio Pineda, Bajo Calima. 
observaciones directas de campo se identificó que el método más utilizado para encontrar el alimento por estas aves fue el de rebuscadores (55\%), seguido por los atrapadores (29\%) y otros con menor abundancia; el estrato de forrajeo donde se alimentan las especies tuvo mayor inclinación por el sotobosque (49\%), seguido por el dosel (26\%) y suelo (14\%).

Se evidenció una alta inclinación hacia el anidamiento individual con el $89 \%$ del total de especies observadas, mientras que solo el $11 \%$ de las especies registradas presentaron un apoyo entre individuos de la misma especie. En relación con el comportamiento social se encontró que el 47 \% participó en bandadas ( $26 \%$ bandada mixta y $21 \%$ bandada monoespecífica), el $42 \%$ de las especies identificadas presentó un comportamiento solitario y un menor porcentaje (10 \%) tuvo un comportamiento social de congregación.

El $82 \%$ del total de especies observadas fueron residentes. Se identificaron 31 especies (18\%) en estatus migratorio, de las cuales 17 especies (10\%) son migratorias locales, 12 especies (7\%) migratorias continentales; finalmente, dos especies (1\%) son migratorias altitudinales, con base en la descripción ecológica de las especies registradas tomadas de López et al. (2016; anexo 1).
El $18 \%$ de las especies registradas anidó y llevó a cabo su fase de reproducción dentro de cavidades que están dispuestas en árboles; de los cuales el $14 \%$ correspondieron a aves que habitan huecos ya existentes dentro de árboles y el 4 \% fueron aves que fabrican sus propias cavidades (anexo 1). Según las categorías de riesgo, en la nación se registró una especie Patagioenas subvinacea (Columbidae) en la en categoría VU (vulnerable) y la especie Tangara johannae (Thraupidae) se encuentra en la categoría LC (bajo riesgo). No se registraron especies en la categoría de riesgo nacional según la resolución n. 1912 de 2017. Según la Unión Internacional para la Conservación de la Naturaleza (UICN), se registraron tres especies en categoría NT (casi amenazada) Ramphastos ambiguus (Ramphastidae), Amazona farinosa (Psittacidae) y Tangara johannae (Thraupidae), tanto en el marco internacional como el nacional. También, se registraron 22 especies casi-endémicas y dos especies de interés para Colombia (Amazilia amabilis y Chalybura ucrochrysia - Trochilidae) (Chaparro et al., 2013; anexo 1).

Con base en la documentación de historia de vida de las especies registradas en las localidades establecidas en los diferentes tipos de hábitat donde se realizaron las observaciones (interior de bosque, área abierta y borde de bosque), se

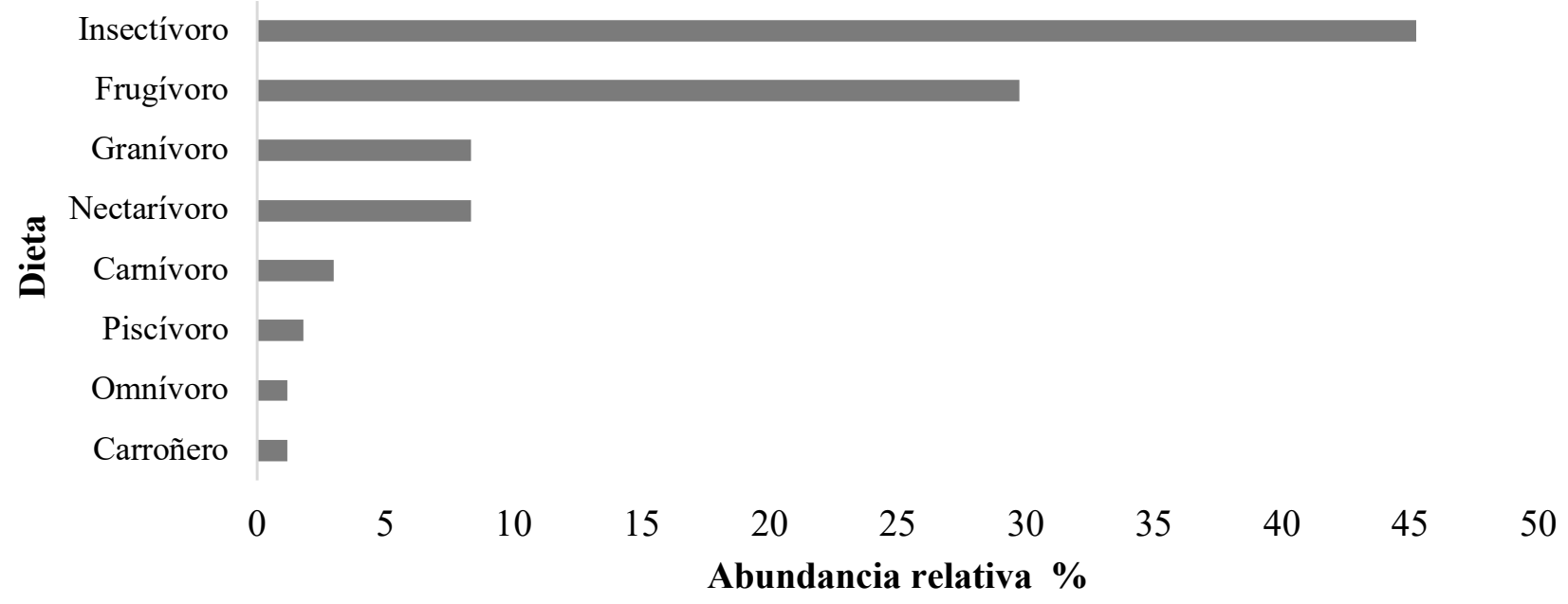

Figura 3. Abundancia relativa de la dieta de las aves presentes en el CFT Pedro Antonio Pineda, Bajo Calima, Colombia. 
identificaron las categorías ecológicas a las cuales corresponde cada especie. Se registró en la categoría ecológica la 20 especies $(11.9 \%)$; lb representada por 33 especies (19.6\%); II fue la más numerosa con 69 especies $(41.1 \%)$; III registró 32 especies (19\%); IVa, tres especies; IVb presentó siete especies; $\mathrm{Va}$ y $\mathrm{Vb}$ registraron dos especies cada una (figura 4, anexo 1).

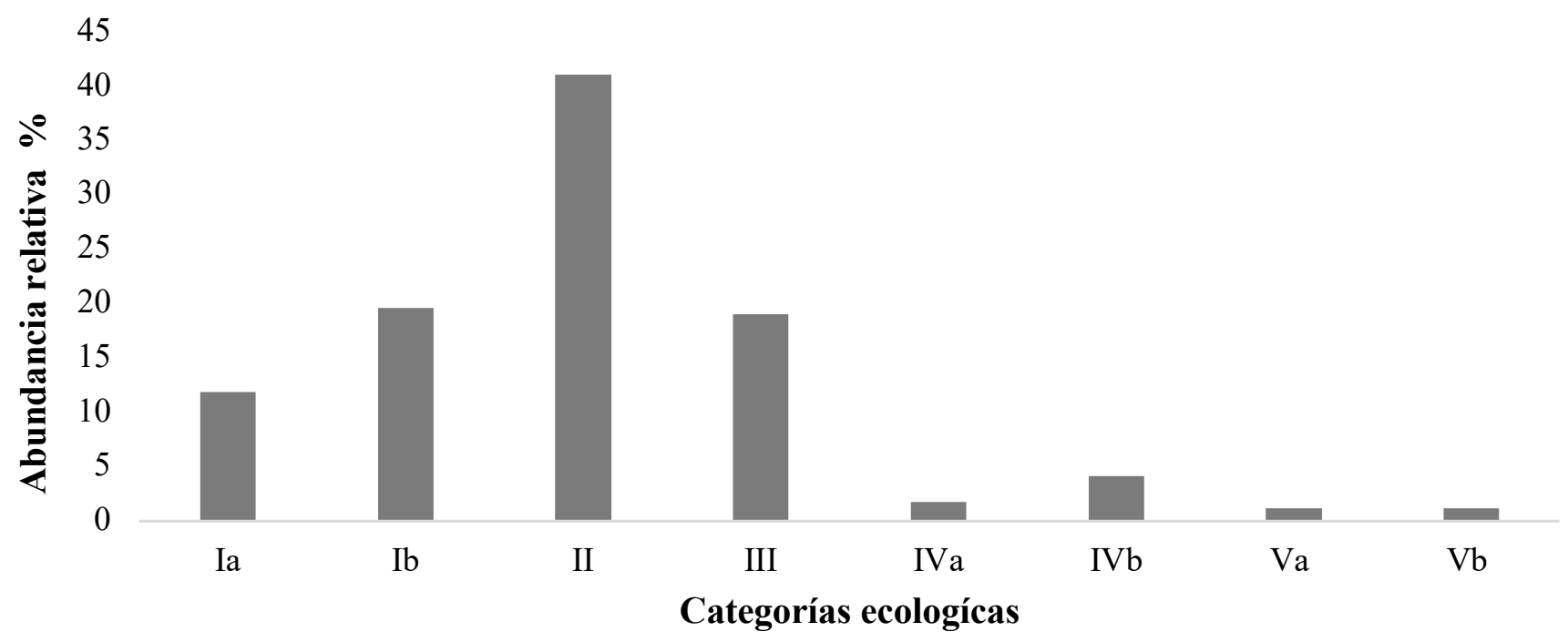

Figura 4. Categorías ecológicas de las especies de aves registradas en el CFT Pedro Antonio Pineda, Bajo Calima, Colombia.

\section{DISCUSIÓN}

El total de aves registradas en los alrededores del CFT Pedro Antonio Pineda, Bajo Calima (166 especies) equivale al $8.6 \%$ del total conocido para Colombia (1932 especies) y al $21.3 \%$ de las especies presentes en la región del Choco biogeográfico, según lo reportado por Rangel (2005; 2015). Este último registró 778 especies de aves para esta región, lo que equivale al $41 \%$ de la riqueza avifaunística del país y al $32 \%$ de las especies registradas en la región Pacífica, de acuerdo con Calderón-Leytón, et al. (2011) para el departamento de Nariño. En la cuenca media del río Calima Castillo-Crespo y Gonzales-Anaya (2007) registraron 320 especies de aves que representan aproximadamente el $32.7 \%$ de las aves registradas para el Valle del Cauca. Para esta misma zona se encuentran reportes con aumento de 522 especies, que actualmente significan el 53.3 \% de las especies (Fundación Trópico y Corporación Autónoma Regional del Valle del Cauca [FT-CARVC], 2017); Quimbayo (2009) reporta resultados inferiores con 85 especies para la misma zona de estudio.

Estos valores de riqueza son una interesante representatividad de aves. teniendo en cuenta que corresponde al $20 \%$ de las especies registradas para el Choco biogeográfico. Estos valores se deben a las características de la estructura del bosque; y a pesar de la presión antrópica asociada a las actividades de explotación tradicional de madera en el área de estudio, estos bosques secundarios se encuentran en un estado de conservación y recuperación mediante un proceso de sucesión natural con una tendencia a la heterogeneidad y variedad de hábitat que se pueden apreciar en más de tres estratos, de igual modo su composición florística puede superar las 70 especies por ha. Se ha señalado que la estratificación tanto horizontal como vertical de la vegetación logra promover la 
coexistencia de un mayor número de especies de aves, ya que estas se benefician tanto de la cobertura protectora como de los recursos alimenticios disponibles (FT-CARVC, 2017).

Según Avendaño (2017), las regiones de la costa Pacífica y el Chocó presentan una concentración de publicaciones sobre la avifauna del $9.1 \%$ y $5.0 \%$, respectivamente, con registros desde los años 1986. No obstante, estos registros corresponden a la región del Pacífico sur y del Chocó-Darién.

La alta representación de las familias Thraupidae y Tyrannidae coincide con los resultados reportados por la FT-CARVC (2017) en el establecimiento de un área protegida en el Alto y Bajo Calima, por Ayerbe et al. (2008) para el departamento del Cauca y Rangel (2004) para el Choco biogeográfico. Tal situación favorece que la mayoría de estas especies se caractericen por ser relativamente "comunes", presentando una amplia distribución geográfica, razón por la cual tienden a ser las más abundantes localmente que aquellas especies que presentan distribución restringida (FT-CARVC, (2017). Esto también se da por las características del hábitat y la disponibilidad de alimento (Peña y Claros, 2016). En este sentido, los gremios tróficos insectívoros y frugívoros fueron los más representativos, dado que los artrópodos son un recurso disponible durante todo el año, en contraste, a las flores, frutos y semillas que son recursos con picos de abundancia marcados durante periodos cortos (FT-CARVC, 2017). Estos resultados coinciden con lo reportado por Quimbayo (2009) para la misma zona de estudio, y por la FT-CARVC (2017) en el establecimiento de un área protegida en el Alto y Bajo Calima.

Por otro lado, las especies casi endémicas registradas representaron el $11 \%$ del total de esta categoría en Colombia (Chaparro et al., 2013). Se destacó Tangara johannae registrada por Quimbayo (2009) en la misma zona de estudio y por la FT-CARVC (2017) en el establecimiento de un área protegida en el Alto y Bajo Calima. Esta especie se encuentra internacionalmente en categoría NT (UICN), mientras que a nivel nacional se encuentra en categoría LC (Rengifo, 2014).

La abundancia relativa de especies en la categoría ecológica II (42%) demostró que el estado del ecosistema representado principalmente por bosques secundarios se mantiene, en contraste con la categoría ecológica la (12.3\%) que es uno de los tipos de cobertura natural que poco se presenta en la zona de estudio. Estos bosques secundarios encontrados en los alrededores del CFT son el resultado del aprovechamiento de la tala rasa realizada entre los decenios de 1970 y 1980 en aproximadamente el 35 \% de este territorio. Están constituidos en su mayoría por árboles de pequeñas dimensiones que no superaron los $30 \mathrm{~cm}$ de diámetro normal y menos $20 \mathrm{~m}$ de altura total y actualmente son la mayor fuente de abastecimiento de madera de la explotación tradicional maderera que realizan las comunidades afrodescendientes. Especies de bosques maduros como chanul, trapichero y chachajo son muy escasas, aunque en las categorías inferiores de la regeneración natural pueden estar presentes. A pesar de la corta edad de recuperación, estos bosques tienden a la heterogeneidad ya que se pueden apreciar más de tres estratos y en cuanto a la composición florística superan las 70 especies por hectárea. En contraste, la ampliación de la frontera agrícola también ejerce presión en el ecosistema natural mediante la implementación de frutales como árbol del pan, zapote, guabos, caimos, anón, borojó, limón y guayabilla, en algunas ocasiones asociados a sistemas agroforestales; otras plantas agrícolas representativas en la zona de estudio son papa china, maíz, arroz, plátano primitivo, yuca y lulo, junto a una gran variedad de plantas medicinales. Además, las comunidades asentadas en este territorio tienen a la fauna silvestre como fuente de proteína a partir de la caza tradicional de especies como guagua (Cuniculus paca), guatín (Dasyprocta punctata), entre otros (Moreno, 2009). A pesar de los diferentes contrastes productivos y culturales sobre el manejo tradicional, estos bosques 
secundarios actúan como hábitat y proveedores de alimento de la mayoría de especies de aves registradas, contando con una interesante representatividad de especies residentes de la zona (82 \%). En relación con las aves migratorias, las 31 especies identificadas en algún estatus migratorio para este estudio son similares a lo reportado por la FT-CARVC (2017), quienes registraron un total de 26 especies de aves migratorias para la zona del Alto y Bajo Calima.

Los bosques del bajo Calima conservan, a pesar del actual deterioro de sus estructuras por la acción antrópica, la composición florística y riqueza de especies de los bosques originales. Lo cual se convierte en un escenario de gran interés para la implementación de estrategias de conservación y manejo sostenible que lleven a la producción de bienes y servicios ecosistémicos, la conservación de áreas forestales y de interés cultural, así como su relación ecológica con las especies de aves, esto con la participación comunitaria que permita por igual el rescate de su patrimonio cultural y biológico.

\section{CONCLUSIONES}

Este registro de las especies de aves del CFT del Bajo Calima es la primera aproximación al conocimiento de la diversidad de fauna silvestre después de 50 años de presencia de la Universidad del Tolima en la región.

A pesar de la presión antrópica asociada a las actividades de explotación tradicional de made$\mathrm{ra}$, estos bosques secundarios se encuentran en estado de recuperación mediante procesos de sucesión natural con una tendencia a la heterogeneidad, ya que se pueden apreciar más de tres estratos y la composición florística puede superar las 70 especies por ha. Estas características estructurales del bosque permiten contar con una interesante representatividad de aves, teniendo en cuenta que corresponde al $20 \%$ de las especies registradas para el Choco biogeográfico y que no se reportan especies en categorías de riesgo en la nación, pero sí actúa como hábitat para 22 especies casi endémicas y para especies que requieren de cavidades de árboles para anidación y el 82 \% de las especies residentes.

Se sugiere establecer actividades de monitoreo de las especies endémicas en: categorías de riesgo, especies en las categorías ecológicas la y lb y especies que cumplen con funciones ecológicas asociadas a los servicios ecosistémicos de regulación (control de insectos, polinización y dispersión de semillas), mediante observaciones periódicas tanto en los bosques secundarios como primarios,

\section{AGRADECIMIENTOS}

Al ingeniero Nelson Javier Albarán, director del CFT Pedro Antonio Pineda, Bajo Calima, de la Universidad del Tolima, por toda la colaboración y el apoyo en las gestiones. Al profesor Omar Melo, por el acompañamiento en algunas de sus prácticas. A la profesora Johanna Magaly García, por la disponibilidad de información de las prácticas de su asignatura Diagnóstico de cuencas. Al ingeniero Julián Leal Villamil, por el apoyo en la cartografía. Y a la comunidad de Las Brisas, por el acompañamiento y apoyo en cada una de las salidas de campo.

\section{CONFLICTO DE INTERESES}

Los autores declaran no tener conflicto de intereses.

\section{CONTRIBUCIÓN POR AUTOR}

Todos los autores planearon y diseñaron la investigación, coordinaron y llevaron a cabo el trabajo en campo y laboratorio, analizaron los datos y redactaron el manuscrito. 


\section{REFERENCIAS}

Andrade, M. G. (2011). Estado del conocimiento de la biodiversidad en Colombia y sus amenazas. Consideraciones para fortalecer la interacción ciencia-política. Revista de la Academia Colombiana de Ciencias Exactas, Físicas y Naturales, 35(137), 491-507.

Armenteras, D. y Rodríguez, N. (2014). Dinámicas y causas de deforestación en bosques de Latino América: una revisión desde 1990. Colombia Forestal, 17(2), 233-246.

https://doi.org/10.14483/udistrital.jour.colomb.for. 2014.2.a07

Avendaño, J. E., Bohórquez, C. I., Rosselli, L., Arzuza-Buelvas, D., Estela, F. A., Cuervo., A. M, Stiles, F. G y Renjifo, L. M. (2017). Lista de chequeo de las aves de Colombia: una síntesis del estado del conocimiento desde Hilty \& Brown (1986). Ornitología Colombiana, 16 (eA01), 1-83.

Ayerbe, F., López, J. P., González, M. F., Estela, F. A., Ramírez, M. B., Sandoval, J. V. y Gómez, L. G. (2008). Aves del departamento del Cauca-Colombia. Biota Colombiana, 9(1), 77-132.

Ayerbe, F. Q. (2018). Guía ilustrada de la avifauna colombiana. Colombia: Wildlife Conservation Society.

Bocanegra, W., Ossa, G. y Melo, O. A. (2009). La región del Bajo Calima. En O. A. Melo y L. A. Lozano (eds.), Los bosques secundarios del trópico húmedo colombiano (p. 139). Ibagué, Colombia: Universidad del Tolima.

Botero, C. A. (2010). El Chocó biogeográfico un tesoro de la naturaleza.

https://www.gestiopolis.com/choco-biogeografico -tesoro-naturaleza/

Calderón-Leytón, J. J., Flórez, C., Cabrera-Finley., A. y Rosero, Y. (2011). Aves del departamento de Nariño, Colombia. Biota Colombiana, 12(1).

Castillo-Crespo, L. S. y González-Anaya, M. (2007). Avances en la implementación del Plan de Acción en Biodiversidad del Valle del Cauca. Agenda de investigación en biodiversidad y vertebrados amenazados. Cali, Colombia: Corporación Autónoma Regional del Valle del Cauca.
Chaparro, S., Echeverry, M. A., Córdoba, S. y Sua, A. (2013). Listado actualizado de las aves endémicas y casi endémicas de Colombia. Biota Colombiana, 14(12), 235-272.

Donegan, T., Quevedo, A., Verhelst J. C., Cortés, O., Ellery, T. y Salaman, P. (2015). Revision of the status of bird species occurring or reported in Colombia 2015, with discussion of BirdLife International's new taxonomy. Conservación Colombiana, 23, 3-48.

Fundación Trópico y Corporación Autónoma Regional del Valle del Cauca (2017). Establecimiento de un área protegida en el Alto y Bajo Calima, área clave de biodiversidad en el Valle del Cauca. Colombia.

García, H. (2014). Deforestación en Colombia: retos y perspectivas. Fedesarrollo.

https://www.repository.fedesarrollo.org.co/handle/ $11445 / 337$

González, P. L., Estades, C. F. y Simonetti, J. A. (2006). Strengthened insectivory in a temperate fragmented forest. Oecología, 148(1), 137-143. https://doi.org/10.1007/s00442-005-0338-3

Hilty, S. L. y Brown, W. L. (1986). A Guide to the Birds of Colombia. Princeton, Nueva Jersey: Princeton University Press.

Instituto de Investigación de Recursos Biológicos Alexander von Humboldt (2012). Informe sobre el estado de los recursos naturales renovables y del ambiente, componente de biodiversidad, 20102011. Bogotá, Colombia: Instituto de Investigación de Recursos Biológicos Alexander von Humboldt.

López, J. P., Stiles F. G. y Parra. J. L. (2016). Protocolo para la medición de rasgos funcionales en aves. En B. Salgado (ed.), La ecología funcional como aproximación al estudio, manejo y conservación de la biodiversidad: protocolos y aplicaciones (p. 236). Bogotá, Colombia: Instituto de Investigación de Recursos Biológicos Alexander von Humboldt.

Lozano, L. A. y González, J. I. (2009). Explotación maderera tradicional de tucas y varas en los bosques secundarios del Bajo Calima. En O. A. Melo y L. A. Lozano (eds.), Los bosques secundarios del trópico húmedo colombiano (p. 139). Ibagué, Colombia: Universidad del Tolima. 
McComb, B. (2008). Wildlife Habitat Management: Concepts and Applications in Forestry. (1 ra ed.). Oregon State University, Corvallis, Oregon, EE. UU.: CRC Press.

https://doi.org/10.1201/9781420007633

McMullan, M., Donegan, T. M. y Quevedo, A. (2010). Guía de campo de las aves de Colombia. Bogotá, Colombia: Fundación ProAves.

Moreno, S. (2009). El pueblo negro del territorio ancestral del Bajo Calima. En O. A. Melo y L. A. Lozano (eds.), Los bosques secundarios del trópico húmedo colombiano (p. 139). Ibagué, Colombia: Universidad del Tolima.

Myers, N., Mittermeier, R. A., Mittermeier, C. G., da Fonseca, G. A. y Kent, J. (2000). Biodiversity hotspots for conservation priorities. Nature, 403(6772), 853-8. https://doi.org/10.1038/35002501

Peña, J. L. y Claros, A. F. (2016). Estudio preliminar de la avifauna en el campus de la Universidad de la Amazonia, en Florencia, Caquetá. Colombia. Revista Biodiversidad Neotropical, 6(1), 85-92. https://doi.org/10.18636/bioneotropical.v6i1.352

Quimbayo, M. A. (2009). Fauna silvestre. En O. A Melo, y L. A. Lozano (eds.), Los bosques secundarios del trópico húmedo colombiano (p. 139). Ibagué, Colombia: Universidad del Tolima.

Rangel, J. O. (2004). Notas sobre la riqueza avifaunística en el Chocó biogeográfico. En J. O. Rangel, (ed.), Colombia diversidad biótica IV. El Chocó biogeográfico/costa Pacífica. Bogotá: Universidad Nacional de Colombia.

Rangel, J. O. (2005). La biodiversidad de Colombia. Palim: Revista de las Facultad de Ciencias Humanas, Universidad Nacional de Colombia, 5, 1657-5083. http://www.raccefyn.co/index.php/raccefyn/article/ view/136

Rangel, J. O. (2015). La biodiversidad de Colombia: significado y distribución regional. Revista de la Academia Colombiana de Ciencias Exactas, Físicas y Naturales, 31(151), 176-200.

https://doi.org/10.18257/raccefyn.136

Renjifo, L. M., Gómez, M. F., Velásquez, J., Amaya, A. M., Kattan, G. H., Amaya, J. D. y Burbano, J. (2014). Libro rojo de aves de Colombia, Volumen I: bosques húmedos de los Andes y la costa Pacífica. Bogotá, Colombia: Pontificia Universidad Javeriana; Instituto de Investigación de Recursos Biológicos Alexander von Humboldt.

Stiles, F. G y Bohórquez, C. I. (2000). Evaluando el estado de la biodiversidad: el caso de la avifauna de la serranía de las Quinchas, Boyacá, Colombia. Caldasia, 22(1), 61-92.

Valoyes, Z., Ramírez, G., Klinger, W. y Carabalí, F. (2012). Componente ecosistémico: identificación y caracterización de la estructura ecológica principal del Chocó biogeográfico según criterio de diversidad y singularidad de especies y ecosistemas. Bioetnia, 9(2), 111-135. 


\section{ANEXO}

Anexo 1. Listado de especies encontradas en el Centro Forestal Tropical "Pedro Antonio Pineda" de la Universidad del Tolima, Bajo Calima, Buenaventura.

\begin{tabular}{|c|c|c|c|}
\hline & Taxón & Nombre común & $\begin{array}{c}\text { Características } \\
\text { ecológicas }\end{array}$ \\
\hline & TINAMIFORMES & & \\
\hline & Tinamidae & & \\
\hline \multirow[t]{3}{*}{1} & Crypturellus berlepschi (Rothschild, 1897) & Tinamú de berlepsch & $\mathrm{Ib}-\mathrm{R}-\mathrm{CE}$ \\
\hline & GALLIFORMES & & \\
\hline & Odontophoridae & & \\
\hline \multirow[t]{3}{*}{2} & Rhynchortyx cinctus (Salvin, 1876) & Colín Carirrufo & $\mathrm{lb}-\mathrm{R}$ \\
\hline & COLUMBIFORMES & & \\
\hline & Columbidae & & \\
\hline 3 & Claravis pretiosa (Ferrari-Pérez, 1886) & Tortolita azulada & II - R \\
\hline 4 & Columba livia (Linnaeus, 1758) & Paloma doméstica & III - I \\
\hline 5 & Columbina talpacoti(Temminck, 1809) & Tortola común/rojiza & III - MC \\
\hline 6 & Geotrygon montana (Linnaeus, 1758) & Paloma cara roja & la $-M L$ \\
\hline 7 & Leptotrygon veraguensis (Lawrence, 1867) & Paloma-perdiz Cariblanca & $\mathrm{R}$ \\
\hline 8 & Patagioenas cayennensis (Bonnaterre, 1792) & Paloma morada & III - R \\
\hline 9 & Patagioenas speciosa (Gmelin, 1789) & Paloma escamosa & $I I-R$ \\
\hline 10 & Patagioenas subvinacea (Lawrence, 1868) & Paloma colorada & $\mathrm{Ib}-\mathrm{R}-\mathrm{VU}$ \\
\hline \multirow[t]{3}{*}{11} & Zenaida auriculata (Des Murs, 1847) & Torcaza naguiblanca & III - ML \\
\hline & CUCULIFORMES & & \\
\hline & Cuculidae & & \\
\hline 12 & Crotophaga major (Gmelin, 1788) & Garrapatero piquiliso & II - R \\
\hline 13 & Crotophaga ani (Linnaeus, 1758) & Garrapatero común & III - R \\
\hline \multirow[t]{3}{*}{14} & Piaya cayana (Linnaeus, 1766) & Cuco ardilla & $\mathrm{Ib}-\mathrm{R}$ \\
\hline & NYCTIBIIFORMES & & \\
\hline & Nyctibiidae & & \\
\hline 15 & Nyctibius aethereus (Wied, 1820) & Bienparado rabilargo & $\mathrm{lb}-\mathrm{R}$ \\
\hline \multirow[t]{3}{*}{16} & Nyctibius griseus (Gmelin, 1789) & Bienparado común & $I I-R$ \\
\hline & CAPRIMULGIFORMES & & \\
\hline & Caprimulgidae & & \\
\hline \multirow[t]{3}{*}{17} & Chordeiles acutipennis (Hermann, 1783) & Chotacabras menor & II - R \\
\hline & APODIFORMES & & \\
\hline & Apodidae & & \\
\hline 18 & Chaetura cinereiventris (Sclater, 1862) & Vencejo ceniciento & $\mathrm{Va}-\mathrm{ML}$ \\
\hline \multirow[t]{2}{*}{19} & Streptoprocne zonaris (Shaw, 1796) & Vencejo acollarado & $\mathrm{Va}-\mathrm{ML}$ \\
\hline & Trochillidae & & \\
\hline 20 & Amazilia tzacatl (De la llave, 1833) & Amazilia colirrufa & III - R \\
\hline 21 & Polyerata amabilis (Gould, 1853) & Colibrí pechiazul & II - R - EI \\
\hline 22 & Polyerata rosenbergi (Boucard, 1895) & Amazilia del chocó & II - R - CE \\
\hline 23 & Chalybura buffonii (Lesson, 1832) & Colibrí de buffon & II - R \\
\hline 24 & Chalybura urochrysia (Gould, 1861) & Colibrí patirrojo & II - R - EI \\
\hline 25 & Discosura conversii (Bourcier \& Mulsant, 1846) & Cola de lira verde & $\mathrm{lb}-\mathrm{R}$ \\
\hline 26 & Florisuga mellivora (Linnaeus, 1758) & Colibrí collarejo/nuca blanca & $I I-R$ \\
\hline 27 & Glaucis aeneus (Lawrecen, 1867) & Ermitaño bronceado & II - R \\
\hline
\end{tabular}




\begin{tabular}{|c|c|c|c|}
\hline & Taxón & Nombre común & $\begin{array}{c}\text { Características } \\
\text { ecológicas }\end{array}$ \\
\hline 28 & Heliothryx barroti (Bourcier, 1843) & Hada coliblanca & $\mathrm{Ib}-\mathrm{R}$ \\
\hline 29 & Phaethornis striigularis (Gould, 1854) & Ermitaño golirrayado & II - R \\
\hline 30 & Phaethornis yaruqui (Bourcier, 1851) & Ermitaño del pacífico & II - R - CE \\
\hline \multirow[t]{3}{*}{31} & Threnetes ruckeri (Bourcier, 1847) & Ermitaño barbudo & $\mathrm{Ib}-\mathrm{R}$ \\
\hline & GRUIFORMES & & \\
\hline & Rallidae & & \\
\hline 32 & Porphyrio martinica (Purple Gallinule) & Pollona azul & $\mathrm{IVb}-\mathrm{R}$ \\
\hline \multirow[t]{3}{*}{33} & Laterallus albigularis (Lawrence, 1861) & Polluela chocoana & $\mathrm{IVb}-\mathrm{R}$ \\
\hline & CHARADRIIFORMES & & \\
\hline & Charadriidae & & \\
\hline \multirow[t]{2}{*}{34} & Charadrius collaris (Vieillot, 1818) & Chorlo de collar & $\mathrm{IVb}-\mathrm{ML}$ \\
\hline & Scolopacidae & & \\
\hline \multirow[t]{3}{*}{35} & Tringa solitaria (Wilson, 1813) & Andarríos solitario & $\mathrm{IVb}-\mathrm{R}$ \\
\hline & SULIFORMES & & \\
\hline & Phalacrocoracidae & & \\
\hline \multirow[t]{3}{*}{36} & Phalacrocorax brasilianus (Gmelin, 1789) & Cormorán neotropical & $\mathrm{IVb}-\mathrm{ML}$ \\
\hline & PELECANIFORMES & & \\
\hline & Ardeidae & & \\
\hline 37 & Ardea alba (Linnaeus, 1758) & Garza blanca & $\mathrm{IVb}-\mathrm{R}$ \\
\hline 38 & Butorides striata (Linnaeus, 1759) & Garcita rayada & $\mathrm{IVb}-\mathrm{ML}$ \\
\hline 39 & Bubulcus ibis (Linnaeus, 1758) & Garcita bueyera & III - ML \\
\hline \multirow[t]{3}{*}{40} & Cochlearius cochlearius (Linnaeus, 1766) & Garza de pico de barco & IVa - R \\
\hline & CATHARTIFORMES & & \\
\hline & Cathartidae & & \\
\hline 41 & Cathartes aura (Linnaeus, 1758) & Guala cabecirroja & $\mathrm{Vb}-\mathrm{MC}$ \\
\hline \multirow[t]{3}{*}{42} & Coragyps atratus (Linnaeus, 1758) & Gallinazo negro & $\mathrm{Vb}-\mathrm{MC}$ \\
\hline & ACCIPITRIFORMES & & \\
\hline & Accipitridae & & \\
\hline 43 & Rupornis magnirostris (Gmelin, 1788) & Gavilán caminero & II - R \\
\hline \multirow[t]{3}{*}{44} & Leucopternis semiplumbeus (Lawrence, 1861) & Halcón semiplumbroso & III - R \\
\hline & STRIGIFORMES & & \\
\hline & Strigidae & & \\
\hline 45 & Ciccaba virgata (Cassin, 1850) & Búho moteado & la $-\mathrm{R}$ \\
\hline \multirow[t]{3}{*}{46} & Megascops choliba (Vieilot, 1817) & Currucutú común & $I I-R$ \\
\hline & TROGONIFORMES & & \\
\hline & Trogonidae & & \\
\hline 47 & Trogon chionurus (Sclater and Salvin, 1871) & Trogón coliblanco occidental & $\mathrm{lb}-\mathrm{R}-\mathrm{CE}$ \\
\hline \multirow[t]{3}{*}{48} & Trogon massena (Gould, 1838) & Trogon grande & $\mathrm{lb}-\mathrm{R}$ \\
\hline & CORACIIFORMES & & \\
\hline & Momotidae & & \\
\hline \multirow[t]{3}{*}{49} & Electron platyrhynchum (Leadbeater, 1829) & Momoto picoancho & la $-\mathrm{R}$ \\
\hline & GALBULIFORMES & & \\
\hline & Bucconidae & & \\
\hline \multirow[t]{3}{*}{50} & Notharchus tectus (Boddaert, 1783) & Bobo coronado & $\| \mathrm{I}-\mathrm{R}$ \\
\hline & PICIFORMES & & \\
\hline & Ramphastidae & & \\
\hline 51 & Ramphastos ambiguus (Swainson, 1823) & Tucán guarumero & $\mathrm{lb}-\mathrm{R}$ \\
\hline 52 & Ramphastos brevis (Meyer de Schauensee, 1945) & Tucán del pacífico & $\mathrm{lb}-\mathrm{R}-\mathrm{CE}$ \\
\hline
\end{tabular}




\begin{tabular}{|c|c|c|c|}
\hline & Taxón & Nombre común & $\begin{array}{c}\text { Características } \\
\text { ecológicas }\end{array}$ \\
\hline 53 & $\begin{array}{l}\text { Pteroglossus torquatus (Gmelin, 1788) } \\
\text { Picidae }\end{array}$ & Pichi collarejo & $\mathrm{Ib}-\mathrm{R}$ \\
\hline 54 & Celeus loricatus (Reichenbach, 1854) & Carpintero canelo & la $-\mathrm{R}$ \\
\hline 55 & Dryocopus lineatus (Linnaeus, 1766) & Carpintero real & II - R \\
\hline 56 & Melanerpes pucherani (Malherbe, 1849) & Carpintero antifaz & II - R \\
\hline 57 & $\begin{array}{l}\text { Veniliornis kirkii (Malherbe, 1845) } \\
\text { FALCONIFORMES } \\
\text { Falconidae }\end{array}$ & Carpintero rabirojo & II - R \\
\hline 58 & $\begin{array}{l}\text { Herpetotheres cachinnans (Linnaeus, 1758) } \\
\text { PSITTACIFORMES } \\
\text { Psittacidae }\end{array}$ & Halcón Guaco & II - R \\
\hline 59 & Amazona farinosa (Boddaert, 1783) & Lora real & III - R \\
\hline 60 & Brotogeris jugularis (Muller, 1776) & Periquito bronceado & $\|-R$ \\
\hline 61 & Pyrilia pulchra (Berlepsch, 1897) & Lorito carirrosado & II - R - CE \\
\hline 62 & $\begin{array}{l}\text { Pionus menstruus (Linnaeus, 1766) } \\
\text { PASSERIFORMES } \\
\text { Thamnophilidae }\end{array}$ & Cotorra cabeciazúl & II - R \\
\hline 63 & Cercomacra nigricans (Sclater, 1858) & Hormiguero yeguá & $\mathrm{lb}-\mathrm{R}$ \\
\hline 64 & Cercomacroides tyrannina (Sclater, 1855) & Hormiguero negruzco/tirano & II - R \\
\hline 65 & Epinecrophylla fulviventris (Lawrence, 1862) & Hormigureo leonado & la $R$ \\
\hline 66 & Gymnopithys bicolor (Lawrence, 1863) & Hormiguero Bicolor & $\mathrm{lb}-\mathrm{R}$ \\
\hline 67 & Myrmotherula pacifica (Hellmayr, 1911) & Hormiguero del pacífico & II - R - CE \\
\hline 68 & Myrmotherula axillaris (Vieillot, 1817) & Hormiguero flanquiblanco & la $-R$ \\
\hline 69 & Microrhopias quixensis (Cornalia, 1849) & Hormiguerito del quijos o abanico & la $-\mathrm{R}$ \\
\hline 70 & Sipia berlepschi (Hartert, 1898) & Hormiguero colimocho & $\mathrm{lb}-\mathrm{R}-\mathrm{CE}$ \\
\hline 71 & Poliocrania exsul (Sclater, 1859) & Hormiguero dorsicastaño & $\mathrm{Ib}-\mathrm{R}$ \\
\hline 72 & Phaenostictus mcleannani (Lawrence, 1860) & Hormiguero ocelado & $\mathrm{lb}-\mathrm{R}$ \\
\hline \multirow[t]{2}{*}{73} & Thamnophilus atrinucha (Salvin \& Godman, 1892) & Batará occidental & $I I-R$ \\
\hline & Furnariidae & & \\
\hline 74 & Automolus ochrolaemus (Tschudi, 1844) & Hojarasquero oliváceo & la $-\mathrm{R}$ \\
\hline 75 & Dendrocincla fuliginosa (Vieillot, 1818) & Trepatronco pardo & la $-\mathrm{R}$ \\
\hline 76 & Glyphorynchus spirurus (Vielliot, 1819) & Trepatronco pico de cuña & la $-M L$ \\
\hline \multirow[t]{2}{*}{77} & Xenerpestes minlosi (Berlepsch, 1886) & Colagrís Norteño & II - R - CE \\
\hline & Tyrannidae & & \\
\hline 78 & Conopias albovittatus (Lawrence, 1862) & Bienteveo del Chocó & $I I-R$ \\
\hline 79 & Colonia colonus (Vieillot, 1818) & Atrapamoscas rabijunco & $\| I-R$ \\
\hline 80 & Elaenia flavogaster (Thunberg, 1822) & Fiofío ventriamarillo & III - R \\
\hline 81 & Legatus leucophaius (Vieillot, 1818) & Atrapamoscas pirata & II - ML \\
\hline 82 & Mionectes olivaceus (Lawrence, 1868 & Mosquerito oliváceo & $\mathrm{la}-\mathrm{R}$ \\
\hline 83 & Mionectes oleagineus (Lichtenstein, 1823) & Atrapamoscas ocráceo & $\mathrm{Ib}-\mathrm{ML}$ \\
\hline 84 & Myiozetetes cayanensis (Linneaus, 1766) & Suelda crestinegra & III - R \\
\hline 85 & Ornithion brunneicapillus (Lawrence, 1862) & Mosquerito coronipardo & $I I-R$ \\
\hline 86 & Todirostrum cinereum (Linneaus, 1766) & Titirijí común & III - R \\
\hline 87 & Todirostrum nigriceps (Sclater, 1855) & Titirijí cabeza negra & $\mathrm{lb}-\mathrm{R}-\mathrm{CE}$ \\
\hline 88 & Tolmomyias assimilis (Pelzeln, 1868) & Pico plano aliamarillo & la $-\mathrm{R}$ \\
\hline 89 & Terenotriccus erythrurus (Cabanis, 1847) & Atrapamoscas colirrufo & II - R \\
\hline 90 & Tyrannulus elatus (Vieillot, 1818) & Mosquerito coronado & $\| I-R$ \\
\hline 91 & Tyrannus melancholicus (Vieillot, 1819) & Sirirí común & III - MA \\
\hline
\end{tabular}




\begin{tabular}{|c|c|c|c|}
\hline & Taxón & Nombre común & $\begin{array}{c}\text { Características } \\
\text { ecológicas }\end{array}$ \\
\hline \multirow[t]{2}{*}{92} & Zimmerius chrysops (Sclater, 1859) & Mosquerito caridorado & II - R \\
\hline & Cotingidae & & \\
\hline 93 & Carpodectes hopkei (Berlepsch, 1897) & Cotinga blanca & III - ML - CE \\
\hline 94 & Lipaugus unirufus (Sclater, 1859) & Guardabosque rufo & II - ML \\
\hline \multirow[t]{2}{*}{95} & Querula purpurata (Mûller, 1776) & Frutero negro - Cuaba & la $-M L$ \\
\hline & Pipridae & & \\
\hline 96 & Lepidothrix coronata (Spix, 1825) & Saltarín coronado & $\mathrm{lb}-\mathrm{R}$ \\
\hline 97 & Manacus manacus (Linnaeus, 1766 & Saltarín barbiblanco & II - R \\
\hline \multirow[t]{2}{*}{98} & Ceratopipra mentalis (Sclater, 1857) & Saltarín cabecirrojo & $\mathrm{lb}-\mathrm{R}$ \\
\hline & Tityridae & & \\
\hline 99 & Pachyramphus cinnamomeus (Lawrence, 1861) & Cabezon canelo & II - R \\
\hline 100 & Tityra semifasciata (Spix, 1825) & Titira enmascarada & II - R \\
\hline \multirow[t]{2}{*}{101} & Tityra inquisitor (Lichtenstein, 1823) & Titira coroninegra & $\| I-R$ \\
\hline & Vireonidae & & \\
\hline 102 & Pachysylvia decurtata (Bonaparte, 1838) & Verderón chico & la $-\mathrm{R}$ \\
\hline \multirow[t]{2}{*}{103} & Vireo olivaceus (Linnaeus, 1766) & Verderón ojirrojo & II - MC \\
\hline & Corvidae & & \\
\hline \multirow[t]{2}{*}{104} & Cyanocorax affinis (Pelzelin, 1856) & Carriqui pechiblanco & II - R - CE \\
\hline & Hirundinidae & & \\
\hline 105 & Hirundo rustica (Linnaeus, 1758 & Golondrina común & III - MC \\
\hline 106 & Atticora tibialis (Cassin, 1853) & Golondrina patiblanca & $\|$ II R \\
\hline 107 & Progne chalybea (Gmelin, 1789) & Golondrina de campanaria & III - MC \\
\hline 108 & Pygochelidon cyanoleuca (Vielliot, 1817) & Golondrina Blanquiazul & III - MC \\
\hline 109 & Riparia riparia (Linnaeus, 1758) & Golondrina Ribereña & III - MC \\
\hline \multirow[t]{2}{*}{110} & Stelgidopteryx ruficollis(Vieillot, 1817) & Golondrina barranquera & III - MC \\
\hline & Troglodytidae & & \\
\hline 111 & Cantorchilus nigricapillus (Sclater, 1860) & Cucarachero ribereño & II - R \\
\hline 112 & Campylorhynchus albobrunneus (Lawrence, 1862) & Cucarachero cabeciblanco & II - R - CE \\
\hline 113 & Cyphorhinus phaeocephalus (Sclater, 1860) & Cucarachero gaitero/canoro & $\mathrm{lb}-\mathrm{R}$ \\
\hline 114 & Henicorhina leucosticta (Cabanis, 1847) & Cucarachero pechiblanco & la $-\mathrm{R}$ \\
\hline 115 & Pheugopedius fasciatoventris (Lafrasnaye, 1845) & Cucarachero ventrinegro & $\mathrm{lb}-\mathrm{R}-\mathrm{CE}$ \\
\hline 116 & Troglodytes aedon (Vielliot, 1809) & Cucarachero común & III - R \\
\hline \multirow[t]{2}{*}{117} & Microcerculus marginatus (Sclater, 1855) & Cucarachero ruiseñor & la $-\mathrm{R}$ \\
\hline & Polioptillidae & & \\
\hline 118 & Microbates cinereiventris (Sclater, 1855) & Curruca rabicunda & $\mathrm{la}-\mathrm{R}$ \\
\hline 119 & Polioptila plumbea (Gmelin, 1788) & Currua Tropical & III - ML \\
\hline \multirow[t]{2}{*}{120} & Polioptila schistaceigula (Hartert, 1898) & Curruca pizarra & $\mathrm{Ib}-\mathrm{R}-\mathrm{CE}$ \\
\hline & Turdidae & & \\
\hline \multirow[t]{2}{*}{121} & Turdus obsoletus (Lawrence, 1862) & Zorzal ventripálido & $\mathrm{la}-\mathrm{R}$ \\
\hline & Passerellidae & & \\
\hline 122 & Chlorospingus flavigularis (Sclater, 1852) & Montero Gorgiamarillo & II - R - CE \\
\hline \multirow[t]{2}{*}{123} & Arremon aurantiirostris (Lafresnaye, 1847) & Pinzón pico de oro & la $R$ \\
\hline & Fringillidae & & \\
\hline 124 & Euphonia fulvicrissa (Sclater, 1857) & Eufonia fulva & II - R - CE \\
\hline 125 & Euphonia minuta (Cabanis, 1849) & Fruterito menudito & II - R \\
\hline \multirow[t]{2}{*}{126} & Euphonia xanthogaster (Sundevall, 1834) & Eufonia buchinaranja & $\mathrm{lb}-\mathrm{R}$ \\
\hline & (2) & & \\
\hline 127 & Psarocolius wagleri (J E Gray, 1845) & Oropéndola cabecicastaña & II - R \\
\hline
\end{tabular}




\begin{tabular}{|c|c|c|c|}
\hline & Taxón & Nombre común & $\begin{array}{c}\text { Características } \\
\text { ecológicas }\end{array}$ \\
\hline 128 & Cacicus cela (Linnaeus, 1758) & Arrendajo culiamarillo & III - R \\
\hline 129 & Cacicus uropygialis (Lafresnaye, 1843) & Arrendajo culirrojo & II - R \\
\hline 130 & Molothrus bonariensis (Gmelin, 1789) & Chamón común/parasito & III - R \\
\hline 131 & Molothrus oryzivorus (Gmelin, 1788) & Tordo gigante & III - R \\
\hline \multirow[t]{2}{*}{132} & Quiscalus mexicanus (Gmelin, 1789) & Chango común & $\mathrm{IVa}-\mathrm{R}$ \\
\hline & Parulidae & & \\
\hline 133 & Parkesia Noveboracensis (Gmelin, 1789) & Reinita charquero & II - MC \\
\hline 134 & Setophaga castanea (Wilson, 1810) & Reinita castaña & II - MC \\
\hline 135 & Setophaga petechia ((Linnaeus, 1766)) & Reinita dorada & III - MC \\
\hline \multirow[t]{2}{*}{136} & Myiothlypis fulvicauda (Spix, 1825) & Reinita guardaribera & IVa - MA \\
\hline & Mitrospingidae & & \\
\hline \multirow[t]{2}{*}{137} & Mitrospingus cassinii (Lawrecen, 1861) & Maraquera carisucia & $\mathrm{lb}-\mathrm{R}$ \\
\hline & Cardinalidae & & \\
\hline \multirow[t]{2}{*}{138} & Cyanoloxia cyanoides (Lafresnaye, 1847) & Azulón silvícola & la - ML \\
\hline & Thraupidae & & \\
\hline 139 & Coereba flaveola (Linneaus, 1758) & Mielero común & II - R \\
\hline 140 & Cyanerpes caeruleus (Linnaeus, 1758) & Mielero ceúleo & $\mathrm{Ib}-\mathrm{R}$ \\
\hline 144 & Cyanerpes cyaneus (Linnaeus, 1766) & Mielero patirrojo & II - R \\
\hline 142 & Chlorophanes spiza (Linnaeus, 1758) & Mielero verde & II - R \\
\hline 143 & Chrysothlypis salmoni (Sclater, 1886) & Tangara escarlata & $\mathrm{Ib}-\mathrm{R}-\mathrm{CE}$ \\
\hline 141 & Dacnis cayana (Linnaeus, 1766) & Dacnis azùl & $\mathrm{lb}-\mathrm{R}$ \\
\hline 145 & Dacnis venusta (Lawrecen, 1862) & Dacnis negriazúl & II - R \\
\hline 146 & Heterospingus xanthopygius (Sclater, 1855) & Chambergo cuernirrojo & II - R -CE \\
\hline 147 & Sicalis flaveola (Linnaeus, 1766) & Canario coronado/costeño & III - R \\
\hline 148 & Volatinia jacarina (Linnaeus, 1766) & Espiguero saltarín & III - R \\
\hline 149 & Islerothraupis luctuosa (d'Orbigny \& Lafresnaye, 1837) & Parlotero aliblanco & $\mathrm{lb}-\mathrm{R}$ \\
\hline 150 & Ramphocelus dimidiatus (Lafresnaye, 1837) & Toche pico de plata & II - R - CE \\
\hline 151 & Ramphocelus flammigerus (Jardine \& Selby, 1833) & Toche enjalmado & II - R \\
\hline 152 & Tachyphonus delatrii (Lafresnaye, 1847) & Parlotero occidental & III - ML \\
\hline 153 & Tachyphonus rufus (Boddaert, 1783) & Parlotero malcasado & II - R \\
\hline 154 & Tersina viridis (Illiger, 1820) & Azulejo golondrina & II - R \\
\hline 155 & Sporophila corvina (P.L sclater, 1859) & Espiguero variable & III - R \\
\hline 156 & Sporophila nigricollis (Vieillot, 1823) & Espiguero capuchino & III - R \\
\hline 157 & Sporophila funerea (Sclater, 1859) & Arrocero piquigrueso & III - R \\
\hline 158 & Saltator grossus (Linnaeus, 1766) & Picogordo pizarra & $\mathrm{Ib}-\mathrm{R}$ \\
\hline 159 & Saltator maximus (Cabanis, 1851) & Saltador ajicero & II - R \\
\hline 160 & Poecilostreptus palmeri (Hellmayr, 1909) & Tangara platinada & II -R - CE \\
\hline 161 & Stilpnia larvata (Du Bus De Gisignies, 1846) & Tangara collareja & II - R \\
\hline 162 & Tangara lavinia (Cassin, 1858) & Tangara cabecirrufa & II - R \\
\hline 163 & Tangara gyrola (Linnaeus, 1758) & Tangara alirrufa & II - R \\
\hline 164 & Tangara johannae (Dalmas, 1900) & Tangara bigotuda & II - R - CE \\
\hline 165 & Thraupis episcopus (Linnaeus, 1766) & Azulejo común & II - R \\
\hline 166 & Thraupis palmarum (Wied, 1821) & Azulejo palmero & II - R \\
\hline
\end{tabular}

Nota. Categorías ecológicas (la, Ib, II, III, IVa, IVb, Va y Vb). Estatus migratorio (R: residente; ML: migración local; MC: migración continental; MA: migración altitudinal) y endemismo (E: endémica; CE: casi endémica; El: especie de interés para Colombia; I: introducida).

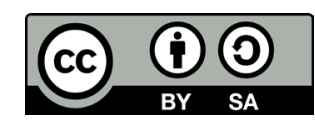

Colombia Forestal •ISSN 0120-0739 • e-ISSN 2256-201X • Bogotá-Colombia • Vol. 23 No. 2 • Julio-diciembre de $2020 \bullet$ pp. 59-74. 